\title{
Asynchronous Stabilization of Switched Singular Systems with Interval Time-Varying Delays
}

\author{
Li-Li Liu, Yue-E Wang and Bao-Wei Wu \\ College of Mathematics and Information Science, \\ Shaanxi Normal University, Xi'an 710062, P R China \\ liulily@snnu.edu.cn,baihewye@163.com,wubw@snnu.edu.cn
}

\begin{abstract}
This paper concerns the asynchronous stabilization problem of switched singular systems with interval time-varying delays. In ideal cases, the stabilizing controller is designed under the assumption that the switching times between the system and the controller are exactly the same. In fact, there are time-delays in the switching signal of the controller, which results in asynchronous switching. The closed-loop system may be unstable under the average dwell time and the controllers derived by ignoring the switching delay. For this case, based on piecewise Lyapunov-Krasovskii functional technique and merging signal method, some sufficient conditions for existence of a stabilizing controller are provided to guarantee that the switched singular time-delay system is regular, impulse free, and exponentially stable with the new average dwell time conditions. Then, the stabilizing controller depending on the asynchronous information is designed. Finally, a numerical example is given to demonstrate the effectiveness of the results.
\end{abstract}

Keywords: Switched singular systems, Time-varying delay, Average dwell time, Exponential stability

\section{Introduction}

This paper investigates the stabilization problem for switched systems composed of a family of linear singular systems with time-varying delays. It's well known that the singular system model is a natural presentation of dynamic systems and it can describe a large class of systems than regular ones such as large scale systems, power system and constrained control systems. The study for singular systems is much more complicated than that for standard state-space systems, as the regularity and absence of impulses (for continuous systems) and causality (for discrete systems) must be considered simultaneously for singular systems [1]. On the other hand, time-delay is a common phenomenon frequently encountered in practical systems, and is often the main cause of instability and poor performance of the dynamical systems [2]. There has been a lot of work reported on stability analysis and synthesis of singular systems and time-delay systems [3-8].

A switched system is a hybrid dynamical system that consists of a finite number of subsystems and a logical rule that orchestrates switching between them [9-11]. The stability issues of switched systems include several interesting phenomena. For example, even when all the subsystems are exponentially stable, the switched systems may have divergent trajectories for certain switching signals. Another remarkable fact is that one may carefully switch between unstable subsystems to make the switched system exponentially stable [12]. As these examples suggest, the stability of switched systems depend not only on the dynamics of each subsystem but also on the properties of switching signals. For switched singular time-delay systems (SSTD), because of the algebraic constraints in singular model and the coupling between the switching and time- 
delay, the behavior of such systems is much more complicated than that of regular switched systems. So far, some appreciable work has been performed on the stability and synthesis for various types of SSTD. In [13], the robust stability and $\mathrm{H} \infty$ control problems for discrete-time uncertain SSTD systems under arbitrary switching were discussed by using the switched Lyapunov function method. In [14], a switching signal was constructed to guarantee the asymptotic stability of continuous-time SSTD systems. In [15], a class of switching signals has been identified for SSTD to be robustly exponentially admissible under the average dwell time scheme. In [16], delay-dependent exponential stability conditions are developed for a class of switched nonlinear singular time-delay systems consisting of stable and unstable subsystems.

Recently, the control issue of switched systems with asynchronous switching signal has drawn increasing attention [17-23]. In many situations, when the switching signal available to the controller is a delayed version of the system's switching, the closed-loop systems can have asynchronous switching. For instance, when the systems and the controller communicate via a communication channel, it requires some time to identify the active system and apply the corresponding controller. For this case, the closed-loop system can experience asynchronous switching signal. Ref. [18] addressed stability of state feedback switched linear systems in which time-delays are present in both the feedback state and the switching signals of the controller. In [20], the issue of asynchronous $\mathrm{H} \infty$ control for a class of switched delay systems was investigated. In [21], the stabilization problem for a class of switched neutral systems was studied. In [22, 23], the regularity, causality and exponential divergence of the singular subsystem were determined by testing the exponential divergence of the transformed linear subsystem. Based on it, the state feedback control problem for a class of discrete-time switched singular systems with time-varying delay under asynchronous switching has been investigated. In this paper, we consider the stabilization problem of continuous-time switching singular systems with time-varying delay under asynchronous switching.

The contribution of this paper lies in three aspects. First, we consider a more general class of switched systems, in which the subsystem is singular systems and time-delay exists in both the state and the controller's switching signal. This class of switched systems covers those considered in [18],[20] and [21] as special cases, where only timeinvariant time-delays or switching delay was considered, or all subsystems were required to be nonsingular; Second, by constructing a new piecewise Lyapunov-Krasovskii functional and using the merging signal method, some sufficient conditions are derived to guarantee the closed-loop system to be exponential admissible. Third, we show that the influence caused by asynchronous switching can be compensated by designing the switching signal satisfying a switching delay-dependent average dwell time scheme.

The organization of the paper is as follows. The problem formulation is stated in Section 2, followed by the main results in Section 3. A numerical example is given in Section 4. Finally, we conclude the paper in Section 5.

Notations: Throughout this paper, the superscript " $T$ " stands for matrix transposition,

$\square^{n}$ denotes the n-dimensional Euclidean space with vector norm $\square \square, \square^{n \times m}$ is the set of all $n \times m$ real matrices, and the notation $P>0$ means that $\mathrm{P}$ is symmetric and positive definite. $\lambda_{\max }(P)$ and $\lambda_{\min }(P)$ denote the maximum and minimum eigenvalues of $P$ respectively. The symmetric elements of the symmetric matrix will be denoted by $*$.

\section{Preliminaries and Problem Formulation}

Consider the switched singular system with time-varying delay described by

$$
\Sigma:\left\{\begin{aligned}
E \dot{x}(t) & =A_{\sigma(t)} x(t)+B_{\sigma(t)} x(t-d(t))+D_{\sigma(t)} u(t) \\
x(t) & =\phi(t), \quad t \in\left[-d_{2}, 0\right] .
\end{aligned}\right.
$$


Where $x(t) \in \mathbb{R}^{n}$ is the state, $u(t) \in \mathbb{R}^{m}$ is the control input. $\phi(t)$ is a compatible vector valued initial function. $\sigma(t):[0, \infty) \rightarrow \mathcal{I}=\{1,2, \cdots, N\}$ with integer $N>1$ is a piecewise constant function of time $t$, called switching signal. Corresponding to $\sigma(t)$, we have the switching sequence,

$$
\left\{x_{t_{0}}:\left(k_{0}, t_{0}\right),\left(k_{1}, t_{1}\right), \cdots,\left(k_{i}, t_{i}\right), \cdots \mid k_{i} \in \mathcal{I}, i \in \mathbb{N}\right\}
$$

which means that the $k_{i}$ th subsystem is active when $t \in\left[t_{i}, t_{i+1}\right)$. The matrix $E \in \mathbb{R}^{n \times n}$ may be singular, we assume that $\operatorname{rank} E=r \leq n . d(t)$ is a time-varying delay that satisfies

$$
d_{1} \leq d(t) \leq d_{2}, \dot{d}(t) \leq \mu
$$

where $d_{1} \geq 0, d_{2}>0$ and $0 \leq \mu<1$ are constants. We assume that no jump occurs in the state at a switching time and that only finitely many switches can occur in any finite interval.

Throughout the paper, we will adopt the following definition.

Definition $1[1,15]$ For the switching signal $\sigma(t)$ and any delay satisfying (1), the system $\Sigma$ with $u(t)=0$ is said to be

(1) regular if $\operatorname{det}\left(s E-A_{i}\right)$ is not identically zero for each $\sigma(t)=k_{i}, k_{i} \in \mathcal{I}$;

(2) impulse free if $\operatorname{deg}\left(\operatorname{det}\left(s E-A_{i}\right)\right)=\operatorname{rank} E$ for each $\sigma(t)=k_{i}, k_{i} \in \mathcal{I}$;

(3) exponentially stable under the switching signal $\sigma(t)$ if the solution $x(t)$ of system $\Sigma$ satisfies

$$
\|x(t)\| \leq c e^{-\lambda\left(t-t_{0}\right)}\left\|x\left(t_{0}\right)\right\|_{d_{2}}, \quad \forall t \geq t_{0}
$$

where $\lambda>0$ and $c>0$ are called the decay rate and decay coefficient respectively;

(4) exponentially admissible if it is regular, impulse free and exponentially stable under the switching signal $\sigma(t)$.

Definition 2 [9] For the switching signal $\sigma(t)$ and any $T_{2}>T_{1} \geq 0$, let $N_{\sigma}\left(T_{1}, T_{2}\right)$ denotes the number of switching of $\sigma(t)$ over $\left(T_{1}, T_{2}\right)$, if $N_{\sigma}\left(T_{1}, T_{2}\right) \leq N_{0}+\frac{T_{2}-T_{1}}{\tau_{a}}$ holds for $\tau_{a}>0, N_{0} \geq 0$, then $\tau_{a}$ is called average dwell time and $N_{0}$ is called a chatter bound.

In this paper, the controller's switching signal is a delayed version of the plant's switching signal, i.e. the control signal going into the plant is

$$
u(t)=K_{\sigma(t-\tau(t))} x(t)
$$

where $\tau(t)$ is the uncertain switching delay satisfying $0 \leq \tau(t) \leq \tau_{d}$. We assume that the maximal switching delay $\tau_{d}$ is known a priori and $\tau_{d} \leq t_{i+1}-t_{i}, i \in \mathcal{I}$. Then the corresponding closed-loop system is given by

$$
\Sigma_{1}:\left\{\begin{aligned}
E \dot{x}(t) & =\left(A_{\sigma(t)}+D_{\sigma(t)} K_{\sigma(t-\tau(t))}\right) x(t)+B_{\sigma(t)} x(t-d(t)), \\
x(t) & =\phi(t), \quad t \in\left[-d_{2}, 0\right] .
\end{aligned}\right.
$$

We will employ the merging signal technique in [18] to deal with the asynchronous switching signal.

Lemma 1 [15] For any positive definite matrix $Q \in \mathbb{R}^{n \times n}$, positive scalar $\alpha$ and vector function $x:[-d, \infty) \rightarrow \mathbb{R}^{n}$ such that the following integration is well defined, then

$$
\int_{t-d(t)}^{t} e^{\alpha(s-t)} x^{T}(s) Q x(s) d s \geq \frac{\alpha}{e^{\alpha d}-1}\left(\int_{t-d(t)}^{t} x(s) d s\right)^{T} Q\left(\int_{t-d(t)}^{t} x(s) d s\right) .
$$

where $t \geq 0,0 \leq d(t) \leq d$. 


\section{Main Results}

In this section, by applying the average dwell time approach and the piecewise Lyapunov functional technique, we present some sufficient conditions and design a state feedback controller to guarantee the exponential admissibility of the SSTD systems $\Sigma$.

Theorem 1. For prescribed scalars $d_{1} \geq 0, d_{2}>0, \tau_{d}>0, \mu<1, \alpha>0, \beta>0$ and $\gamma \geq 1$, suppose that there exist matrices $\bar{P}, \bar{Q}_{k_{i} k_{j}}, \bar{R}_{k_{i} k_{j}}$ and $\bar{M}_{k_{i}}, \forall k_{i}, k_{j} \in \mathcal{I}$, such that

$$
\begin{aligned}
& E \bar{P}=\bar{P}^{T} E^{T} \geq 0, \\
& \Phi=\left[\begin{array}{ccc}
\Phi_{11} & B_{k_{i}} \bar{P} & 0 \\
* & -(1-\mu) e^{-\alpha d_{2}} \bar{Q}_{k_{i} k_{i}} & 0 \\
* & * & -e^{-\alpha d_{1}} \bar{R}_{k_{i} k_{i}}
\end{array}\right]<0, \\
& \Psi=\left[\begin{array}{ccccc}
\Psi_{11} & B_{k_{i}} \bar{P} & 0 & 0 & 0 \\
* & -(1-\mu) e^{-\alpha d_{2}} \bar{Q}_{k_{i} k_{j}} & 0 & 0 & 0 \\
* & * & -e^{-\alpha d_{1}} \bar{R}_{k_{i} k_{j}} & 0 & 0 \\
* & * & * & -c_{1} \bar{R}_{k_{i} k_{j}} & 0 \\
* & * & * & * & -c_{2} \bar{Q}_{k_{i} k_{j}}
\end{array}\right]<0, \\
& \bar{Q}_{k_{i} k_{j}} \leq \gamma \bar{Q}_{k_{m} k_{n}}, \bar{R}_{k_{i} k_{j}} \leq \gamma \bar{R}_{k_{m} k_{n}}, k_{i}, k_{j}, k_{m}, k_{n} \in \mathcal{I},
\end{aligned}
$$

where $\Phi_{11}=A_{k_{i}} \bar{P}+\bar{P}^{T} A_{k_{i}}^{T}+D_{k_{i}} M_{k_{i} k_{i}}+M_{k_{i} k_{i}}^{T} D_{k_{i}}^{T}+\alpha E \bar{P}+\bar{Q}_{k_{i} k_{i}}+\bar{R}_{k_{i} k_{i}}$,

$$
\begin{aligned}
& \Psi_{11}=A_{k_{i}} \bar{P}+\bar{P}^{T} A_{k_{i}}^{T}+D_{k_{i}} M_{k_{j}}+M_{k_{j}}^{T} D_{k_{i}}^{T}-\beta E \bar{P}+\bar{Q}_{k_{i} k_{j}}+\bar{R}_{k_{i} k_{j}}, \\
& c_{1}=\frac{(\alpha+\beta) * \alpha}{e^{\alpha d_{1}}-1}, c_{2}=\frac{(\alpha+\beta) * \alpha}{e^{\alpha d_{2}-1}} .
\end{aligned}
$$

Then the controller (2) can guarantee that system $\Sigma$ is exponential admissibility for any switching signal with average dwell time satisfying

$$
\tau_{a}>\tau_{a}^{*}=\frac{2 \ln \gamma+(\alpha+\beta) \tau_{d}}{\alpha} .
$$

Moreover, the controller gains are given by $K_{i_{k}}=M_{i_{k}} \bar{P}^{-1}$.

Proof: Due to the switching delay, when the $k_{j}$ th subsystem has been switched to the $k_{i}$ th subsystem, the controller $K_{k_{j}}$ still active when $t \in\left[t_{i}, t_{i}+\tau\left(t_{i}\right)\right), i \in \mathcal{I}$. Thus the closed-loop subsystem $\left(\Sigma_{1}\right)$ can be embodied as, for all $k_{i}, k_{j} \in \mathcal{I}, k_{i} \neq k_{j}$

$$
\Sigma_{2}: E \dot{x}(t)= \begin{cases}\left(A_{k_{0}}+D_{k_{0}} K_{k_{0}}\right) x(t)+B_{k_{0}} x(t-d(t)), & t \in\left[t_{0}, t_{1}\right) \\ \left(A_{k_{i}}+D_{k_{i}} K_{k_{j}}\right) x(t)+B_{k_{i}} x(t-d(t)), & t \in\left[t_{i}, t_{i}+\tau\left(t_{i}\right)\right) \\ \left(A_{k_{i}}+D_{k_{i}} K_{k_{i}}\right) x(t)+B_{k_{i}} x(t-d(t)), & t \in\left[t_{i}+\tau\left(t_{i}\right), t_{i+1}\right)\end{cases}
$$

We create a virtual switching signal $\tilde{\sigma}(t):[0, \infty) \rightarrow \mathcal{I} \times \mathcal{I}$ as $\tilde{\sigma}(t)=(\sigma(t), \sigma(t-\tau(t)))$. The merging action is denoted by $\ominus$ such that $\tilde{\sigma}(t)=\sigma(t) \oplus \sigma(t-\tau(t))$. From the definition, it follows that the set of switching times of $\tilde{\sigma}(t)$ is the union of the sets of switching times of $\sigma(t)$ and $\sigma(t-\tau(t))$.

The proof of this Theorem is divided into three parts. First of all, we show that the switched singular system is regular and impulse free. Since $\operatorname{rank} E=r \leq n$, there exist nonsingular matrices $M, N \in \mathbb{R}^{n \times n}$ such that $M E N=\operatorname{diag}\left\{I_{r}, 0\right\}$. In this paper, without loss of generality, let $E=\operatorname{diag}\left\{E_{r}, 0\right\}$. and denote $\bar{A}_{k_{i} k_{j}}=A_{k_{i}}+D_{k_{i}} K_{k_{j}}$. We assume that the matrices $\bar{A}_{k_{i} k_{j}}, \bar{P}^{-1}$ have the partitioned matrix forms 


$$
\bar{A}_{k_{i} k_{j}}=\left[\begin{array}{ll}
\bar{A}_{k_{i} k_{j} 11} & \bar{A}_{k_{i} k_{j} 12} \\
\bar{A}_{k_{i} k_{j} 21} & \bar{A}_{k_{i} k_{j} 22}
\end{array}\right], \bar{P}^{-1}=\left[\begin{array}{cc}
P_{11} & P_{12} \\
P_{21} & P_{22}
\end{array}\right],
$$

then (3) implies that $P_{12}=0 . \Psi_{11}<0$ and $\Phi_{11}<0$ derive that $\bar{A}_{k_{i} k_{j} 22} P_{22}+P_{22}^{T} \bar{A}_{k_{i} k_{j} 22}^{T}<0$, which implies that $\bar{A}_{k_{i} k_{j} 22}$ is nonsingular for $k_{i}=k_{j}$ or $k_{i} \neq k_{j}$. By [3] and Definition 1, closed-loop system $\left(\Sigma_{1}\right)$ is regular and impulse free.

Next, we will show the differential subsystem of the closed - loop system $\left(\Sigma_{1}\right)$ is exponentially stable. Define the piecewise Lyapunov functional candidate for system $\left(\Sigma_{1}\right)$ as follows:

$$
\begin{aligned}
V\left(x_{t}\right)= & x^{T}(t) E^{T} P x(t)+\int_{t-d(t)}^{t} e^{\alpha(s-t)} x^{T}(s) Q_{\tilde{\sigma}} x(s) d s \\
& +\int_{t-d_{1}}^{t} e^{\alpha(s-t)} x^{T}(s) R_{\tilde{\sigma}} x(s) d s
\end{aligned}
$$

where $P^{-1}=\bar{P}, Q_{\tilde{\sigma}}=P^{T} \bar{Q}_{\tilde{\sigma}} P, R_{\tilde{\sigma}}=P^{T} \bar{R}_{\tilde{\sigma}} P$,

$$
\tilde{\sigma}=\left\{\begin{array}{l}
k_{0}, \quad t \in\left[t_{0}, t_{1}\right) ; \\
k_{i} k_{j}, \quad t \in\left[t_{i}, t_{i}+\tau\left(t_{i}\right)\right) ; \\
k_{i} k_{i}, \quad t \in\left[t_{i}+\tau\left(t_{i}\right), t_{i+1}\right) .
\end{array}\right.
$$

Then, along the trajectories of $\left(\Sigma_{2}\right)$ for $\tilde{\sigma}=k_{i} k_{i}$, we have

$$
\begin{aligned}
& \dot{V}_{k_{i} k_{i}}\left(x_{t}\right)+\alpha V_{k_{i} k_{i}}\left(x_{t}\right) \\
\leq & 2 x^{T}(t) P^{T} E \dot{x}(t)+x^{T}(t) Q_{k_{i} k_{i}} x(t) \\
& -(1-\mu) e^{-\alpha d_{2}} x^{T}(t-d(t)) Q_{k_{i} k_{i}} x(t-d(t))+x^{T}(t) R_{k_{i} k_{i}} x(t) \\
& -e^{-\alpha d_{1}} x^{T}\left(t-d_{1}\right) R_{k_{i} k_{i}} x\left(t-d_{1}\right)+\alpha x^{T}(t) E^{T} P x(t) \\
= & \xi^{T}(t) \bar{\Phi} \xi(t)
\end{aligned}
$$

Where

$$
\begin{gathered}
\bar{\Phi}=\left[\begin{array}{ccc}
\bar{\Phi}_{11} & P^{T} B_{k_{i}} & 0 \\
* & -(1-\mu) e^{-\alpha d_{2}} Q_{k_{i} k_{i}} & 0 \\
* & * & -e^{-\alpha d_{1}} R_{k_{i} k_{i}}
\end{array}\right], \\
\bar{\Phi}_{11}=P^{T} \bar{A}_{k_{i} k_{i}}+\bar{A}_{k_{i} k_{i}}^{T} P+\alpha E^{T} P+Q_{k_{i} k_{i}}+R_{k_{i} k_{i}}, \\
\xi(t)=\left[\begin{array}{lll}
x^{T}(t) & x^{T}(t-d(t)) & x^{T}\left(t-d_{1}\right)
\end{array}\right]^{T} .
\end{gathered}
$$

Right and left multiplying $\bar{\Phi}$ by $\operatorname{diag}\{\bar{P}, \bar{P}, \bar{P}\}$ and it's transposition, and letting $K_{k_{i}} \bar{P}=M_{k_{i}}$, then we have that $\Phi<0$ implies $\dot{V}_{k_{i} k_{i}}\left(x_{t}\right)+\alpha V_{k_{i} k_{i}}\left(x_{t}\right) \leq 0$.

Similarly, along the trajectories of $\left(\Sigma_{2}\right)$ for $\tilde{\sigma}=k_{i} k_{j}, k_{j}=k_{i-1}$, we have

$$
\begin{aligned}
& \dot{V}_{k_{i} k_{j}}\left(x_{t}\right)-\beta V_{k_{i} k_{j}}\left(x_{t}\right) \\
\leq & 2 x^{T}(t) P^{T} E \dot{x}(t)+x^{T}(t) Q_{k_{i} k_{j}} x(t) \\
& -(1-\mu) e^{-\alpha d_{2}} x^{T}(t-d(t)) Q_{k_{i} k_{j}} x(t-d(t))+x^{T}(t) R_{k_{i} k_{j}} x(t) \\
& -e^{-\alpha d_{1}} x^{T}\left(t-d_{1}\right) R_{k_{i} k_{j}} x\left(t-d_{1}\right)-\beta x^{T}(t) E^{T} P x(t) \\
& -(\alpha+\beta) \int_{t-d_{1}}^{t} e^{\alpha(s-t)} x^{T}(s) R_{k_{i} k_{j}} x(s) d s \\
& -(\alpha+\beta) \int_{t-d(t)}^{t} e^{\alpha(s-t)} x^{T}(s) Q_{k_{i} k_{j}} x(s) d s .
\end{aligned}
$$

It follows Lemma 1, 


$$
\begin{aligned}
\int_{t-d_{1}}^{t} e^{\alpha(s-t)} x^{T}(s) R_{k_{i} k_{j}} x(s) d s & \geq \frac{\alpha}{e^{\alpha d_{1}}-1}\left(\int_{t-d_{1}}^{t} x(s) d s\right)^{T} R_{k_{i} k_{j}}\left(\int_{t-d_{1}}^{t} x(s) d s\right), \\
\int_{t-d(t)}^{t} e^{\alpha(s-t)} x^{T}(s) Q_{k_{i} k_{j}} x(s) d s & \geq \frac{\alpha}{e^{\alpha d_{2}}-1}\left(\int_{t-d(t)}^{t} x(s) d s\right)^{T} Q_{k_{i} k_{j}}\left(\int_{t-d(t)}^{t} x(s) d s\right) .
\end{aligned}
$$

Consider above inequalities, we can obtain

$$
\dot{V}_{k_{i} k_{j}}\left(x_{t}\right)-\beta V_{k_{i} k_{j}}\left(x_{t}\right) \leq \eta^{T}(t) \bar{\Psi} \eta(t) .
$$

Where

$$
\begin{gathered}
\bar{\Psi}=\left[\begin{array}{ccccc}
\bar{\Psi}_{11} & P^{T} B_{k_{i}} & 0 & 0 & 0 \\
* & -(1-\mu) e^{-\alpha d_{2}} Q_{k_{i} k_{j}} & 0 & 0 & 0 \\
* & * & -e^{-\alpha d_{1}} R_{k_{i} k_{j}} & * & 0 \\
* & * & * & -c_{1} R_{k_{i} k_{j}} & * \\
* & * & * & * & -c_{2} Q_{k_{i} k_{j}}
\end{array}\right], \\
\eta(t)=\left[\begin{array}{ccccc}
x^{T}(t) & x^{T}(t-d(t)) & x^{T}\left(t-d_{1}\right) & \left(\int_{t-d(t)}^{t} x(s) d s\right)^{T} & \left(\int_{t-d_{1}}^{t} x(s) d s\right)^{T}
\end{array}\right] .
\end{gathered}
$$

Right and left multiplying $\bar{\Psi}$ by $\operatorname{diag}\{\bar{P}, \bar{P}, \bar{P}, \bar{P}, \bar{P}\}$ and it's transposition, then we have that $\Psi<0$ implies $\dot{V}_{k_{i} k_{j}}\left(x_{t}\right)-\beta V_{k_{i} k_{j}}\left(x_{t}\right) \leq 0$.

From (6), it is obtained on the switching points

$$
V_{k_{i} k_{i}}\left(t_{i}+\tau\left(t_{i}\right)\right) \leq \gamma V_{k_{i} k_{j}}\left(\left(t_{i}+\tau\left(t_{i}\right)\right)^{-}\right), \quad V_{k_{i} k_{j}}\left(t_{i}\right) \leq \gamma V_{k_{j} k_{j}}\left(\left(t_{i}\right)^{-}\right) .
$$

So, for any $t \in\left[t_{i}, t_{i}+\tau\left(t_{i}\right)\right)$, we have

$$
\begin{aligned}
& V_{k_{i} k_{j}}(t) \\
\leq & e^{\beta\left(t-t_{i}\right)} V_{k_{i} k_{j}}\left(t_{i}\right) \\
\leq & \gamma e^{\beta\left(t-t_{i}\right)} V_{k_{j} k_{j}}\left(t_{i}^{-}\right) \\
\leq & \gamma e^{\beta\left(t-t_{i}\right)} e^{-\alpha\left(t_{i}-\left(t_{i-1}+\tau\left(t_{i-1}\right)\right)\right.} V_{k_{j} k_{j}}\left(t_{i-1}+\tau\left(t_{i-1}\right)\right) \\
\leq & \gamma e^{\beta\left(t-t_{i}\right)} e^{-\alpha\left(t_{i}-\left(t_{i-1}+\tau_{d}\right)\right.} V_{k_{j} k_{j}}\left(t_{i-1}+\tau\left(t_{i-1}\right)\right) \\
\leq & \gamma^{2} e^{\beta\left(t-t_{i}\right)} e^{-\alpha\left(t_{i}-\left(t_{i-1}+\tau_{d}\right)\right.} V_{k_{j} k_{j-1}}\left(\left(t_{i-1}+\tau\left(t_{i-1}\right)\right)^{-}\right) \\
\leq & \cdots \\
\leq & \gamma^{2 i-1} e^{i \beta \tau} e^{-\alpha\left(t-t_{0}-i \tau_{d}\right)} V_{k_{0}}\left(t_{0}\right) \\
\leq & \frac{1}{\gamma} e^{\left[2 \ln \gamma+(\alpha+\beta) \tau_{d}\right] N_{0}} e^{\left[\frac{2 \ln \gamma+(\alpha+\beta) \tau_{d}}{T_{a}}-\alpha\right]\left(t-t_{0}\right)} V_{k_{0}}\left(t_{0}\right) .
\end{aligned}
$$

The last inequality follows from that $N\left[t_{0}, t\right]=i \leq N_{0}+\frac{t-t_{0}}{\tau_{a}}$.

Similarly, for any $t \in\left[t_{i}+\tau\left(t_{i}\right), t_{i+1}\right)$, we have

$$
\begin{aligned}
& V_{k_{i} k_{i}}(t) \\
\leq & e^{-\alpha\left(t-\left(t_{i}+\tau\left(t_{i}\right)\right)\right.} V_{k_{i} k_{i}}\left(t_{i}+\tau\left(t_{i}\right)\right) \\
\leq & \gamma e^{-\alpha\left(t-\left(t_{i}+\tau_{d}\right)\right.} V_{k_{i} k_{j}}\left(\left(t_{i}+\tau\left(t_{i}\right)\right)^{-}\right) \\
\leq & \cdots \\
\leq & \gamma^{2 i} e^{i \beta \tau_{d}} e^{-\alpha\left(t-t_{0}-i \tau_{d}\right)} V_{k_{0}}\left(t_{0}\right) \\
\leq & e^{\left[2 \ln \gamma+(\alpha+\beta) \tau_{d}\right] N_{0}} e^{\left[\frac{2 \ln \gamma+(\alpha+\beta) \tau_{d}}{T_{1}}-\alpha\right]\left(t-t_{0}\right)} V_{k_{0}}\left(t_{0}\right) .
\end{aligned}
$$

According to $V_{\tilde{\sigma}}(t)$, we obtain

$$
\lambda_{1}\left\|x_{1}(t)\right\|^{2} \leq V_{\tilde{\sigma}}(t), V_{\tilde{\sigma}}\left(t_{0}\right) \leq \lambda_{2}\left\|x_{t_{0}}\right\|^{2},
$$


where $\lambda_{1}=\min _{k_{i} k_{j} \in \mathcal{I} \times \mathcal{I}} \lambda_{\min }\left(P_{11}\right)$,

$$
\lambda_{2}=\max _{k_{i} k_{j} \in \mathcal{I} \times \mathcal{I}} \lambda_{\max }\left(P_{11}\right)+\frac{1-e^{-\alpha d_{1}}}{\alpha} \max _{k_{i} k_{j} \in \mathcal{I} \times \mathcal{I}} \lambda_{\max }\left(R_{k_{i} k_{j}}\right)+\frac{1-e^{-\alpha d_{2}}}{\alpha} \max _{k_{i} k_{j} \in \mathcal{I} \times \mathcal{I}} \lambda_{\max }\left(Q_{k_{i} k_{j}}\right) .
$$

Then, it yields

$$
\left\|x_{1}(t)\right\| \leq \sqrt{\frac{\lambda_{2}}{\lambda_{1}}} e^{\left[\ln \gamma+(\alpha+\beta) \frac{\left.\tau_{d}\right]}{2}\right] N_{0}} e^{\left[\frac{\left[\ln \gamma+(\alpha+\beta) \tau_{d}\right.}{2 T_{\alpha}}-\frac{\alpha}{2}\right]\left(t-t_{0}\right)}\left\|x_{t_{0}}\right\| .
$$

Denote $\bar{c}=\sqrt{\frac{\lambda_{2}}{\lambda_{1}}} e^{\left[\ln \gamma+(\alpha+\beta) \frac{\tau_{d}}{2}\right] N_{0}}, \bar{\lambda}=\alpha-\left[\frac{2 \ln \gamma+(\alpha+\beta) \tau_{d}}{\tau_{a}}\right]$, i.e. $\left\|x_{1}(t)\right\| \leq \bar{c} e^{-\frac{1}{2} \bar{\lambda}\left(t-t_{0}\right)}$, So, for any switching signals with average dwell time $\tau_{a} \geq \tau_{a}^{*}$, the differential subsystem is exponentially stable.

At last, exponential stability of the algebraic subsystem will be confirmed. As $\bar{A}_{k_{i} k_{j} 22}$ is nonsingular, set

$$
G_{k_{i} k_{j}}=\left[\begin{array}{cc}
I_{r} & -\bar{A}_{k_{i} k_{j} 12} \bar{A}_{k_{i} k_{j} 22}^{-1} \\
0 & \bar{A}_{k_{i} k_{j} 22}^{-1}
\end{array}\right], \hat{P}_{k_{i} k_{j}}=G_{k_{i} k_{j}}^{-T} P=\left[\begin{array}{cc}
\hat{P}_{k_{i} k_{j} 11} & 0 \\
\hat{P}_{k_{i} k_{j} 21} & \hat{P}_{k_{i} k_{j} 22}
\end{array}\right],
$$

and denote

$$
\hat{A}_{k_{i} k_{j}}=G_{k_{i} k_{j}} \bar{A}_{k_{i} k_{j}}=\left[\begin{array}{cc}
\hat{A}_{k_{i} k_{j} 11} & 0 \\
\hat{A}_{k_{i} k_{j} 21} & I_{n-r}
\end{array}\right], \hat{B}_{k_{i}}=G_{k_{i} k_{j}} B_{k_{i}}=\left[\begin{array}{cc}
\hat{B}_{k_{i} k_{j} 11} & \hat{B}_{k_{i} k_{j} 12} \\
\hat{B}_{k_{i} k_{j} 21} & \hat{B}_{k_{i} k_{j} 22}
\end{array}\right] .
$$

Then, system $\Sigma_{2}$ is restricted system equivalent to

$$
\begin{aligned}
\dot{x}_{1}(t) & =\hat{A}_{k_{i} k_{j} 11} x_{1}(t)+\hat{B}_{k_{i} k_{j} 11} x_{1}(t-d(t))+\hat{B}_{k_{i} k_{j} 12} x_{1}(t-d(t)), \\
-x_{2}(t) & =\hat{A}_{k_{i} k_{j} 21} x_{1}(t)+\hat{B}_{k_{i} k_{j} 21} x_{1}(t-d(t))+\hat{B}_{k_{i} k_{j} 22} x_{1}(t-d(t)) .
\end{aligned}
$$

By $\bar{\Psi}<0$ and $\bar{\Phi}<0$, it holds

$$
\left[\begin{array}{cc}
\hat{P}_{k_{i} k_{j} 22}^{T}+\hat{P}_{k_{i} k_{j} 22}+\hat{Q}_{k_{i} k_{j} 22} & \hat{P}_{k_{i} k_{j} 22}^{T} \hat{B}_{k_{i} k_{j} 22} \\
* & -(1-\mu) e^{-\alpha d_{2}} \hat{Q}_{k_{i} k_{j} 22}
\end{array}\right]<0,
$$

Then according to [24], we can deduce that there exist constants $h_{i j}>1$ and $\eta_{i j}>0$ such that

$$
\left\|\left(\hat{B}_{k_{i} k_{j} 22}\right)^{l}\right\| \leq h_{i j} e^{-\left(\eta_{i j}+\frac{1}{2} \alpha d_{2}\right) l} .
$$

For time-varying delay, motivated from [16], some new variables are defined to model the dependency of $x_{2}(t)$ on past instants. Define

$$
t^{0}=t, t^{j}=t^{j-1}-\tau\left(t^{j-1}\right), j=1,2, \cdots .
$$

When $t \in\left[t_{i}+\tau\left(t_{i}\right), t_{i+1}\right)$, there exist integer $N_{k_{i} k_{i}}$ such that $t^{N_{k_{i} k_{i}}} \in\left[t_{i}, t_{i}+\tau\left(t_{i}\right)\right)$ and $t^{N_{k_{i} k_{i}}-1} \in\left[t_{i}+\tau\left(t_{i}\right), t_{i+1}\right)$. By iteration, we have

$$
\begin{aligned}
x_{2}(t)= & \left(-\hat{B}_{k_{i} k_{i} 22}\right)^{N_{k_{i} k_{i}}} x_{2}\left(t^{N_{k_{i} k_{i}}}\right)-\sum_{l_{k_{i} k_{i}}=0}^{N_{k_{i} k_{i}}-1}\left(-\hat{B}_{k_{i} k_{i} 22}\right)^{l_{k_{i} k_{i}}} \times \\
& {\left[\hat{A}_{k_{i} k_{i} 21} x_{1}\left(t^{l_{k_{i} k_{i}}}\right)+\hat{B}_{k_{i} k_{i} 21} x_{1}\left(t^{l_{k_{i} k_{i}}+1}\right)\right] . }
\end{aligned}
$$

Then, following a similar procedure as the above, there exists positive integer $N_{k_{i} k_{i-1}}$ such that $t^{\left(N_{k_{i} k_{i}}+N_{k_{i} k_{i-1}}\right)} \in\left[t_{i-1}+\tau\left(t_{i-1}\right), t_{i}\right)$ and $t^{N_{k_{i} k_{i}}+N_{k_{i} k_{i-1}-1}} \in\left[t_{i}, t_{i}+\tau\left(t_{i}\right)\right)$. By iteration, we have 
International Journal of Control and Automation

Vol.8, No.11 (2015)

$$
\begin{aligned}
x_{2}\left(t^{N_{k_{i} k_{i}}}\right)= & \left(-\hat{B}_{k_{i} k_{i-1} 22}\right)^{\left(N_{k_{i} k_{i-1}}\right)} x_{2}\left(t^{\left(N_{k_{i} k_{i}}+N_{k_{i} k_{i-1}}\right)}\right) \\
& -\sum_{N_{k_{i} k_{i}}+N_{k_{i} k_{i-1}}-1}^{l_{l_{k_{i} k_{i-1}}}=N_{k_{i} k_{i}}}\left(-\hat{B}_{k_{i} k_{i-1} 22}\right)^{l_{k_{i} k_{i}-1}} \times\left[\hat{A}_{k_{i} k_{i-1} 21} x_{1}\left(t^{l_{k_{i} k_{i}-1}}\right)\right] \\
& -\sum_{l_{k_{i} k_{i-1}}=N_{k_{i} k_{i}}}^{N_{k_{i} k_{i}}+N_{k_{i} k_{i-1}}-1}\left(-\hat{B}_{k_{i} k_{i-1} 22}\right)^{l_{k_{i} k_{i-1}}} \times\left[\hat{B}_{k_{i} k_{i-1} 21} x_{1}\left(t^{\left(l_{k_{i} k_{i-1}}+1\right)}\right)\right] .
\end{aligned}
$$

After $2 i$ times iterative manipulations, there exists a finite positive integer $N_{k_{0}}$ such that $t^{\left(N_{k_{i} k_{i}}+N_{k_{i} k_{i-1}}+\cdots+N_{k_{1} k_{0}}+N_{k_{0}}\right)} \in\left[-d_{2}, t_{0}\right)$ and $t^{\left(N_{k_{i} k_{i}}+N_{k_{i} k_{i-1}}+\cdots+N_{k_{1} k_{0}}+N_{k_{0}}-1\right)} \in\left[t_{0}, t_{1}\right)$.

By an induction, we have

$$
\begin{aligned}
& x_{2}(t)=\left(\hat{B}_{k_{0} 22}\right)^{N_{k_{0}}}\left[\prod_{p=1}^{i}\left(\hat{B}_{k_{p} k_{p} 22}\right)^{N_{k_{p} k_{p}}}\left(\hat{B}_{k_{p} k_{p-1} 22}\right)^{N_{k_{p} k_{p}-1}}\right] x_{2}\left(t^{N_{k_{i} k_{i}}+\cdots+N_{k_{1} k_{0}}+N_{k_{0}}}\right) \\
& \sum_{l_{k_{i} k_{i}}=0}^{N_{k_{i} k_{i}}-1}\left(-\hat{B}_{k_{i} k_{i} 22}\right)^{l_{k_{i} k_{i}}} \times\left[\hat{A}_{k_{i} k_{i} 21} x_{1}\left(t^{l_{k_{i} k_{i}}}\right)+\hat{B}_{k_{i} k_{i} 21} x_{1}\left(t^{l_{k_{i} k_{i}}+1}\right)\right] \\
& -\left(-\hat{B}_{k_{i} k_{i} 22}\right)^{N_{k_{i} k_{i}}}\left\{\sum_{l_{k_{i} k_{i}-1}=N_{k_{i} k_{i}}}^{N_{k_{i} k_{i}}+N_{k_{i} k_{i}-1}-1}\left(-\hat{B}_{k_{i} k_{i-1} 22}\right)^{l_{k_{i} k_{i}-1}}\right. \\
& \left.\times\left[\hat{A}_{k_{i} k_{i-1} 21} x_{1}\left(t^{l_{k_{i} k_{i-1}}}\right)+\hat{B}_{k_{i} k_{i-1} 21} x_{1}\left(t^{\left(l_{k_{i} k_{i-1}}+1\right)}\right)\right]\right\}
\end{aligned}
$$

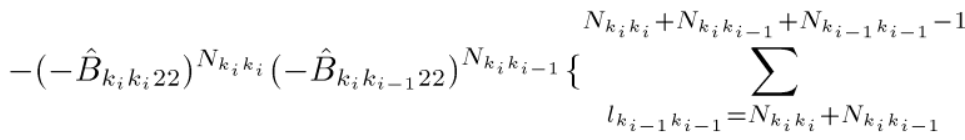

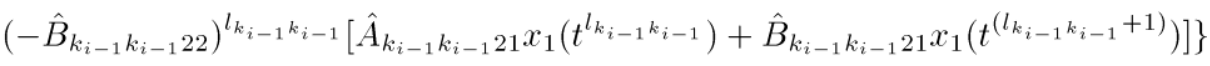

$$
\begin{aligned}
& +\cdots+\left[\prod_{p=1}^{i}\left(\hat{B}_{k_{p} k_{p} 22}\right)^{N_{k_{p} k_{p}}}\left(\hat{B}_{k_{p} k_{p-1} 22}\right)^{N_{k_{p} k_{p}-1}}\right]
\end{aligned}
$$

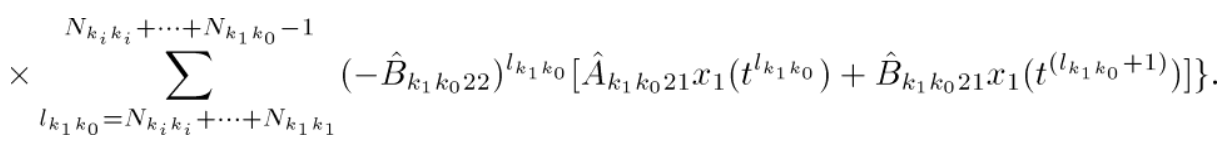

Then 


$$
\begin{aligned}
& \left\|x_{2}(t)\right\| \leq\left\|\left(\hat{B}_{k_{0} 22}\right)^{N_{k_{0}}}\right\|\left[\prod_{p=1}^{i}\left\|\left(\hat{B}_{k_{p} k_{p} 22}\right)^{N_{k_{p} k_{p}}}\left(\hat{B}_{k_{p} k_{p-1} 22}\right)^{N_{k_{p} k_{p-1}}}\right\|\right]\left\|x_{t_{0}}\right\|_{d_{2}} \\
& +\hat{A}_{21} \sum_{l_{k_{i} k_{i}=0}}^{N_{k_{i} k_{i}}-1}\left\|\left(-\hat{B}_{k_{i} k_{i} 22}\right)^{l_{k_{i} k_{i}}}\right\|\left\|x_{1}\left(t^{l_{k_{i} k_{i}}}\right)\right\| \\
& +\hat{B}_{21} \sum_{l_{k_{i} k_{i}}=0}^{N_{k_{i} k_{i}}-1}\left\|\left(-\hat{B}_{k_{i} k_{i} 22}\right)^{l_{k_{i} k_{i}}}\right\|\left\|x_{1}\left(t^{l_{k_{i} k_{i}}+1}\right)\right\| \\
& \sum_{l_{k_{i} k_{i}-1}=N_{k_{i} k_{i}}}^{N_{k_{i} k_{i}}+N_{k_{i} k_{i-1}}-1}\left[\left\|\left(-\hat{B}_{k_{i} k_{i} 22}\right)^{N_{k_{i} k_{i}}}\right\|\left\|\left(-\hat{B}_{k_{i} k_{i-1} 22}\right)^{l_{k_{i} k_{i-1}}}\right\|\left\|x_{1}\left(t^{l_{k_{i} k_{i-1}}}\right)\right\|\right] \\
& +\hat{B}_{21} \sum_{l_{k_{i} k_{i}-1}=N_{k_{i} k_{i}}}^{N_{k_{i} k_{i}}+N_{k_{i} k_{i-1}}-1}\left[\left\|\left(-\hat{B}_{k_{i} k_{i} 22}\right)^{N_{k_{i} k_{i}}}\right\|\left\|\left(-\hat{B}_{k_{i} k_{i-1} 22}\right)^{l_{k_{i} k_{i-1}}}\right\|\left\|x_{1}\left(t^{l_{k_{i} k_{i-1}}+1}\right)\right\|\right] \\
& +\cdots \\
& +\prod_{p=1}^{i}\left\|\left(\hat{B}_{k_{p} k_{p} 22}\right)^{N_{k_{p} k_{p}}}\right\|\left\|\left(\hat{B}_{k_{p} k_{p-1} 22}\right)^{N_{k_{p} k_{p-1}}}\right\| \\
& \times \sum_{l_{k_{1} k_{0}}=N_{k_{i} k_{i}}+\cdots+N_{k_{1} k_{1}}}^{N_{k_{i} k_{i}}+\cdots+N_{k_{1} k_{0}}-1}\left[\hat{A}_{21}\left\|\left(-\hat{B}_{k_{1} k_{0} 22}\right)^{l_{k_{1} k_{0}}}\right\|\left\|x_{1}\left(t^{l_{k_{1} k_{0}}}\right)\right\|\right. \\
& \left.+\hat{B}_{21} \hat{B}_{k_{p} k_{q} 21}\|\|\left(-\hat{B}_{k_{1} k_{0} 22}\right)^{l_{k_{1} k_{0}}}\|\| x_{1}\left(t^{l_{k_{1} k_{0}}+1}\right) \|\right] \text {, }
\end{aligned}
$$

where $\hat{A}_{21}=\max _{k_{p} k_{q} \in \mathcal{I} \times \mathcal{I}}\left\|\hat{A}_{k_{p} k_{q} 21}\right\|, \hat{B}_{21}=\max _{k_{p} k_{q} \in \mathcal{I} \times \mathcal{I}}\left\|\hat{B}_{k_{p} k_{q} 21}\right\|$.

Notice that $\left\|\left(\hat{B}_{k_{p} k_{p-1} 22}\right)^{l}\right\| \leq h_{k_{p} k_{p-1}} e^{-\left(\eta_{k_{p} k_{p-1}}+\frac{1}{2} \alpha d_{2}\right) l}$ and $\left\|x_{1}(t)\right\| \leq \bar{c} e^{-\frac{1}{2} \bar{\lambda}\left(t-t_{0}\right)}$, we have

$$
\begin{aligned}
& \left\|x_{2}(t)\right\| \leq \prod_{p=0}^{p=i} h_{k_{p} k_{p}} h_{k_{p} k_{p-1}} e^{-\left(\eta_{k_{p} k_{p}} N_{k_{p} k_{p}}+\eta_{k_{p} k_{p-1}} N_{k_{p} k_{p-1}}\right)} \bar{c} e^{-\frac{1}{2} \bar{\lambda}\left(t-t_{0}\right)}\left\|x_{t_{0}}\right\|_{d_{2}} \\
& +\hat{A}_{21} \sum_{l_{k_{i} k_{i}=0}}^{N_{k_{i} k_{i}}-1} h_{k_{i} k_{i}} e^{-\left(\eta_{k_{i} k_{i}}+\frac{1}{2} \alpha d_{2}\right) l_{k_{i} k_{i}} \bar{c}} e^{-\frac{1}{2} \bar{\lambda}\left(t^{l} k_{i} k_{i}-t_{0}\right)}\left\|x_{t_{0}}\right\|_{d_{2}} \\
& +\hat{B}_{21} \sum_{l_{k_{i} k_{i}=0}}^{N_{k_{i} k_{i}}-1} h_{k_{i} k_{i}} e^{-\left(\eta_{k_{i} k_{i}}+\frac{1}{2} \alpha d_{2}\right) l_{k_{i} k_{i}} \bar{c}} e^{-\frac{1}{2} \bar{\lambda}\left(t^{l_{k_{i} k_{i}}+1}-t_{0}\right)}\left\|x_{t_{0}}\right\|_{d_{2}} \\
& +\hat{A}_{21} \sum_{l_{k_{i} k_{i}-1}=N_{k_{i} k_{i}}}^{N_{k_{i} k_{i}}+N_{k_{i} k_{i-1}}-1}\left[h_{k_{i} k_{i}} e^{-\left(\eta_{k_{i} k_{i}}+\frac{1}{2} \alpha d_{2}\right) N_{k_{i} k_{i}}} h_{k_{i} k_{i-1}}\right. \\
& \times e^{-\left(\eta_{k_{i} k_{i-1}}+\frac{1}{2} \alpha d_{2}\right) l_{k_{i} k_{i-1}}} \bar{c} e^{-\frac{1}{2} \bar{\lambda}\left(t^{l_{k_{i}} k_{i-1}}-t_{0}\right)}\left\|x_{t_{0}}\right\|_{d_{2}} \\
& +\hat{B}_{21} \sum_{l_{k_{i} k_{i}-1}=N_{k_{i} k_{i}}}^{N_{k_{i} k_{i}}+N_{k_{i} k_{i-1}}-1}\left[h_{k_{i} k_{i}} e^{-\left(\eta_{k_{i} k_{i}}+\frac{1}{2} \alpha d_{2}\right) N_{k_{i} k_{i}}} h_{k_{i} k_{i-1}}\right. \\
& \times e^{-\left(\eta_{k_{i} k_{i-1}}+\frac{1}{2} \alpha d_{2}\right) l_{k_{i} k_{i-1}}} \bar{c} e^{-\frac{1}{2} \bar{\lambda}\left(t^{l_{k_{i}} k_{i-1}+1}-t_{0}\right)}\left\|x_{t_{0}}\right\|_{d_{2}} \\
& +\cdots
\end{aligned}
$$




$$
\begin{aligned}
& +\prod_{p=1}^{p=i} h_{k_{p} k_{p}} h_{k_{p} k_{p-1}} e^{-\left(\eta_{k_{p} k_{p}} N_{k_{p} k_{p}}+\eta_{k_{p} k_{p-1}} N_{k_{p} k_{p}-1}\right)} \times \\
& \sum_{N_{k_{i} k_{i}}+\cdots+N_{k_{1} k_{0}}-1} h_{k_{1} k_{0}} e^{-\left(\eta_{k_{1} k_{0}}+\frac{1}{2} \alpha d_{2}\right) l_{k_{1} k_{0}} \bar{c}} \\
& \times\left[\hat{A}_{21} e^{-\frac{1}{2} \bar{\lambda}\left(t^{\left.l_{k_{1}} k_{0}-t_{0}\right)}+\cdots+N_{k_{1} k_{1}}\right.}+\hat{B}_{21} e^{-\frac{1}{2} \bar{\lambda}\left(t^{l_{k_{1}} k_{0}+1}-t_{0}\right)}\right]\left\|x_{t_{0}}\right\|_{d_{2}} \\
\leq & \hat{c} e^{-\frac{1}{2} \bar{\lambda}\left(t-t_{0}\right)}\left\|x_{t_{0}}\right\|_{d_{2}} .
\end{aligned}
$$

When $t \in\left[t_{i}, t_{i}+\tau\left(t_{i}\right)\right)$, similar procedure can be performed to get same result, which confirms exponential stability of the algebraic subsystem. This completes the proof.

Remark 1 It is worth pointing out that if $E$ is nonsingular, then $\Sigma$ is the normal switched time-delay systems. Our research method is also applied with a little alter.

Remark 2 From the proof, we can see that the Lyapunov - Krasovskii functional is required to decrease only during the controller is match to the active subsystem, and the Lyapunov - Krasovskii functional can increase on the mismatching time interval. It shows that the influence caused by asynchronous switching can be compensated by designing the switching signal satisfying a switching delay - dependent average dwell time scheme.

Remark 3 Some new variables are defined to model the dependency of algebraic sub - state on past instants, and a iterative equation is derived which plays an important role in analyzing the exponential stability of algebraic subsystem.

When the switching delay $\tau(t)=0$, the controller's switching and the systems's switching is synchronous, we have the following corollary.

Corollary 1 For prescribed scalars $d_{1} \geq 0, d_{2}>0, \tau_{d}>0, \mu<1, \alpha>0$ and $\gamma \geq 1$, Suppose that there exist matrices $\bar{P}, \bar{Q}_{k_{i}}, \bar{R}_{k_{i}}$ and $\bar{M}_{k_{i}}, \forall k_{i} \in \mathcal{I}$, such that

$$
\begin{gathered}
\Phi=\left[\begin{array}{ccc}
\Phi_{11} & B_{k_{i}} \bar{P} & E \bar{P}=\bar{P}^{T} E^{T} \geq 0 \\
* & -(1-\mu) e^{-\alpha d_{2}} \bar{Q}_{k_{i}} & 0 \\
* & * & -e^{-\alpha d_{1}} \bar{R}_{k_{i}}
\end{array}\right]<0 \\
\text { where } \Phi_{11}=A_{k_{i}} \bar{P}+\bar{P}^{T} A_{k_{i}}^{T} \leq \gamma \bar{Q}_{k_{j}}, \bar{R}_{k_{i}} \leq \gamma \bar{R}_{k_{j}}, \\
\left.c_{1}=\frac{\alpha+\beta}{\alpha}\left(e^{\alpha d_{1}}-1\right), c_{k_{i}}+M_{k_{i}}^{T} D_{k_{i}}^{T}+\alpha E \bar{P}+\alpha \bar{P}^{\alpha d_{2}}-1\right) .
\end{gathered}
$$

Then, the controller (2) can guarantee that system $(\Sigma)$ is exponential admissibility for any switching signal with average dwell time satisfying

$$
\tau_{a}>\tau_{a}^{*}=\frac{\ln \gamma}{\alpha} .
$$

Moreover, the controller gains are given by $K_{k_{i}}=M_{k_{i}} \bar{P}^{-1}$.

\section{Numerical Examples}

Consider the following switched singular time-delay system, composed of two subsystems described by

Subsystem 1:

$$
E=\left[\begin{array}{ll}
1 & 0 \\
0 & 0
\end{array}\right], A_{1}=\left[\begin{array}{cc}
-5 & 0 \\
0 & 1
\end{array}\right], B_{1}=\left[\begin{array}{cc}
-1 & 1 \\
0 & -0.5
\end{array}\right], D_{1}=\left[\begin{array}{c}
-0.1 \\
0.5
\end{array}\right]
$$

Subsystem 2,

$$
E=\left[\begin{array}{ll}
1 & 0 \\
0 & 0
\end{array}\right], A_{2}=\left[\begin{array}{ll}
-4 & 0 \\
-1 & 1
\end{array}\right], B_{2}=\left[\begin{array}{cc}
-1 & 0.1 \\
0 & -0.1
\end{array}\right], D_{2}=\left[\begin{array}{c}
-0.2 \\
0.2
\end{array}\right] .
$$


For

$\alpha=0.5, \beta=0.1, \mu=0.1, d(t)=0.65+0.5 \sin (0.1 t), d_{1}=0.05, d_{2}=1.15, \gamma=1.15$.

Solving the condition in Theorem 1, we can obtain the minimum ADT $\tau_{a}^{*}=1.1590$ and the corresponding controller gains are

$$
K_{1}=\left[\begin{array}{ll}
-1.1439 & 3.1786
\end{array}\right], K_{2}=\left[\begin{array}{ll}
0.7216 & 3.4228
\end{array}\right] .
$$

\section{Conclusions}

In this paper, the state feedback problem for a class of switched singular timedelay systems under asynchronous switching has been investigated. Delaydependent stability conditions have been derived for the closed-loop system to be regular, impulse free and exponentially stable in the presence of asynchronous switching. The corresponding controller can be determined by solving a set of linear matrix inequalities. With the help of the average dwell time approach, a class of switching signals has been found under which the switched singular time-delay system is exponentially stable. An example has been given to demonstrate the applicability and effectiveness of the proposed approach.

\section{Acknowledgements}

This study was supported by the National Natural Science Foundation of China (11201277) and the Fundamental Research Funds for the Central Universities (GK201102023).

\section{References}

[1] L. Dai, "Singular Control Systems", Springer-Verlag, Berlin (1989).

[2] K. Gu, V. L. Kharitonov and J. Chen, "Stability of Time-delay Systems", Springer-Verlag, New York (2003).

[3] S. Xu and J. Lam, "Robust Control and Filtering of Singular Systems", Springer-Verlag, Berlin (2006).

[4] B. Haidar and E. K. Boukas, "Exponential stability of singular systems with multiple time-varying delays", Automatica, 45(2): 539-545 (2009).

[5] Z. G. Wu, J. H. Park, H. Su, B. Song and J. Chu, "Mixed H and passive filtering for singular systems with time delays", Signal Processing, 93(4): 1705-1711 (2013).

[6] S. Xu, J. Lam, B. Zhang and Y. Zou, "A new result on the delay-dependent stability of discrete systems with time-varying delays", International Journal of Robust and Nonlinear Control, 24(16): 2512-2521 (2014).

[7] P. Shi, E. K. Boukas and R. K. Agarwal, "Control of Markovian jump discrete-time systems with norm bounded uncertainty and unknown delay", IEEE Transactions on Automatic Control, 44(11): 2139-2144 (1999).

[8] M. Basin, J. Rodriguez-Gonzalez and R. Martinez-Zuniga, "Optimal control for linear systems with time delay in control input", Journal of the Franklin Institute, 341(3): 267-278 (2004).

[9] D. Liberzon, "Switching in Systems and Control", Springer-Verlag, Berlin, Genmany (2003).

[10] J. Zhao and D. J. Hill, "On stability, L2-gain and Ho control for switched systems", Automatica, 44(5): 1220-1232 (2008).

[11] X. M. Sun and W. Wang, "Integral input-to-state stability for hybrid delayed systems with unstable continuous dynamics", Automatica, 48(9): 2359-2364 (2012).

[12] H. Lin and P. J. Antsaklis, "Stability and stabilization of switched linear systems: a survey of recent results", IEEE Transations on Automatic Control, 54(2): 308-322 (2009).

[13] S. P. Ma, C. H. Zhang and Z. Wu, "Delay-dependent stability and $\mathrm{H} \infty$ control for uncertain discrete switched singular systems with time-delay", Applied Mathematics and Computation, 206(1): 413-424 (2008).

[14] T. C. Wang and Z. R. Gao, "Asympototic stability criterion for a class of switched uncertain descriptor systems with time-delay", Acta Automatica Sinica, 34(8):1013-1016 (2008).

[15] J. X. Lin and S. M. Fei, "Robust exponential admissibility of uncertain switched singular time-delay systems", Acta Automatica Sinica, 36(12): 1773-1779 (2010). 
[16] I. Zamani, M. Shafiee and A. Ibeas, "Stability analysis of hybrid switched nonlinear singular time-delay systems with stable and unstable subsystems", International Journal of Systems Science, 45(5): 11281144 (2014).

[17] L. X. Zhang and H. J. Gao, "Asynchronously switched control of switched linear systems with average dwell time", Automatica, 46(5): 953-958 (2010).

[18] L. Vu and M. A. Kristi, "Stability of time-delay feedback switched linear systems", IEEE Transactions on Automatic Control, 55(10): 2385-2389 (2010).

[19] Z. R. Xiang, C. Y. Liang and Q. W. Chen, "Robust L2-L filtering for switched systems under asynchronous switching", Communications in Nonlinear Science and Numerical Simulation, 16(8): 3303-3318 (2011).

[20] Y. E. Wang, X. M. Sun and J. Zhao, "Asynchronous $H \infty$ control of switched delay systems with average dwell time", Journal of the Franklin Institute, 349: 3159-3169 (2012).

[21] Y. E. Wang, J. Zhao and B. Jiang, "Stabilization of a class of switched linear neutral systems under asynchronous switching", IEEE Transactions on Automatic Control, 58(8): 2114-2119 (2013).

[22] J. X. Lin, S. M. Fei and Z. F. Gao, "Control discrete-time switched singular systems with state delays under asynchronous switching", International Journal of Systems Science, 44(6): 1089-1101 (2013).

[23] J. X. Lin, S. M. Fei and Z. F. Gao, "Stabilization of discrete-time switched singular time-delay systems under asynchronous switching", Journal of the Franklin Institute, 349: 1808-1827 (2012).

[24] I. R. Peterson, "A stabilization algorithm for a class uncertain linear systems", System Control Letters, 8 : 351-357 (1987).
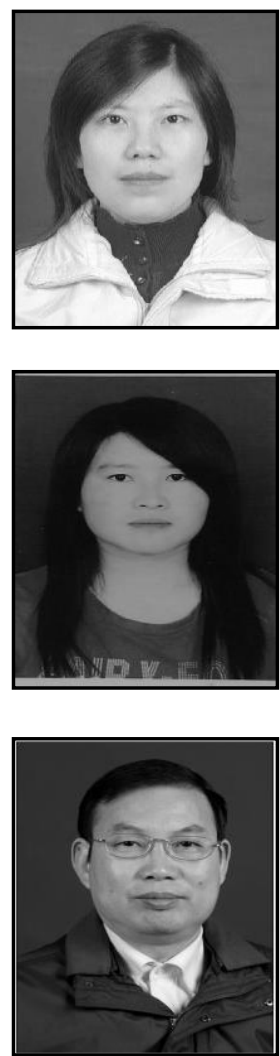

\section{Authors}

Li-Li LIU received her M.S. degree in Applied Mathematics from Shaanxi Normal University, Xi'an, China, in 2005 and the Ph.D. degree in Applied Mathematics from Xi'an Jiaotong University, China, in 2011. She is currently an Associate Professor in College of Mathematics and Information Science from Shaanxi Normal University, Xi'an, China. Her research interests include singular systems, switched systems and time delay systems.

Yue-E Wang received her M.S. degree in College of Mathematics and Information Science from Shaanxi Normal University, Xi'an, China, in 2010, and the Ph.D. degree in Control Theory and Control Engineering from Northeastern University, China, in 2014. She is currently a Lecturer in College of Mathematics and Information Science from Shaanxi Normal University, Xi'an, China. Her research interests include switched systems and time delay systems.

Bao-Wei WU received the Ph.D. degree from Xi'an Jiaotong University in 1998. He is now a full Professor at the College of Mathematics and Information Science, Shaanxi Normal University, Xi'an, China. His main research interests include linear systems, robust control and time-delay systems. 